La mesure des excitations par d'autres techniques expérimentales 
Collection SFN 10 (2010) 467-485

(C) Owned by the authors, published by EDP Sciences, 2010

DOI: $10.1051 / \mathrm{sfn} / 2010009$

\title{
Spectroscopie optique : les vibrations et les phonons
}

\author{
F. Guillaume et M. Couzi
}

\author{
Université Bordeaux 1, UMR CNRS 5255, ISM, Groupe Spectroscopie Moléculaire, \\ 351 cours de la Libération, 33405 Talence Cedex, France
}

\begin{abstract}
Résumé. The aim of this course is to introduce some basic concepts of Raman and infrared spectroscopic techniques. In a first section we describe the interaction of a dielectric material with the electric field to emphasize the fundamental differences between the mechanism of absorption (resonance) for infrared spectroscopy and of scattering (oscillating dipole) for Raman spectroscopy. We thus provide some keys for applying methods based on the Group Theory that allow predicting and analyzing optical phonons at the Brillouin zone center.
\end{abstract}

\section{INTRODUCTION}

L'objectif de ce cours est de montrer la complémentarité existant entre la spectroscopie de diffusion neutronique et les méthodes optiques infrarouge et Raman. Nous rappelons dans une première partie les vibrations attendues dans un cristal atomique ou moléculaire. En spectroscopie optique, la longueur d'onde du rayonnement excitateur est grande par rapport aux dimensions des mailles élémentaires de sorte que seuls les phonons en centre de zone pourront être observés. La réponse d'un matériau diélectrique soumis à la perturbation de la composante électrique du champ extérieur est la polarisation. Nous décrivons les différents mécanismes de polarisation permettant d'introduire les techniques spectroscopiques. En particulier la spectroscopie d'absorption infrarouge est basée sur le phénomène de résonance entre le champ excitateur et les vibrations moléculaires alors que la spectroscopie Raman est basée sur l'émission de l'onde produite par le dipôle oscillant induit par le champ excitateur et modulé par les vibrations. Il résulte de ces mécanismes d'interaction des règles de sélection spécifiques liées aux propriétés de symétrie de l'objet étudié. Ces règles de sélection, qui n'existent pas en diffusion neutronique, sont un atout considérable pour l'attribution des phonons optiques. Nous introduirons donc brièvement les concepts issus de la théorie des groupes permettant de prédire l'activité des modes vibrationnels dans un cristal. La spectroscopie optique est une instrumentation de «paillasse», facilement disponible dans les laboratoires ou au sein de plateformes [1] et pour laquelle des formations sont mises en place depuis longtemps. Les progrès récents dans les domaines de l'optique, de la détection et des lasers ont ouvert la voie vers des applications particulièrement originales et uniques, que ce soit par exemple pour des études d'objets isolés de dimensions nanométriques ou des systèmes chiraux.

\section{LES VIBRATIONS DANS UN CRISTAL}

\subsection{La zone de Brillouin}

\subsubsection{Le cristal atomique}

Rappelons que lorsque l'on considère une maille cristalline constituée de $\mathrm{N}$ atomes, $3 \mathrm{~N}$ degrés de liberté (selon les trois directions de l'espace dans le repère du cristal) sont attendus. Parmi ces $3 \mathrm{~N}$ degrés de

This is an Open Access article distributed under the terms of the Creative Commons Attribution-Noncommercial License 3.0, which permits unrestricted use, distribution, and reproduction in any noncommercial medium, provided the original work is properly cited. 
liberté, 3 correspondent à des déplacements en phase et de même amplitude de tous les atomes de la maille selon les trois directions. Ainsi, les déplacements de chaque maille du cristal selon une direction cristallographique correspondent aux phonons acoustiques (de fréquence nulle en centre de zone de Brillouin), et les relations de phase entre ces déplacements correspondent à la dispersion de la branche dans la zone de Brillouin. Les 3N-3 autres degrés de liberté des atomes dans la maille correspondront aux phonons optiques. Ainsi les courbes de dispersion pour un cristal à $\mathrm{N}$ atomes par maille présenteront 3 branches acoustiques de fréquences nulles à l'origine (en centre de zone) et $3 \mathrm{~N}-3$ branches optiques.

\subsubsection{Le cristal moléculaire}

Considérons maintenant un cas légèrement plus complexe d'un cristal moléculaire de $\sigma$ molécules identiques par maille, chaque molécule étant composée de $\mathrm{N}$ atomes. $3 \mathrm{~N} \sigma$ degrés de libertés sont donc attendus pour chaque maille dont 3 correspondent aux branches acoustiques. Il est judicieux à ce stade de faire la distinction en modes internes et vibrations de réseau. Les modes internes correspondent aux vibrations des molécules ( $3 \mathrm{~N}$ - vibrations par molécule) et les vibrations de réseau aux translations ( $3 \sigma-3$ degrés de liberté) et rotations/librations ( $3 \sigma$ degrés de libertés) des molécules dans la maille. $\mathrm{Au}$ total, nous attendons donc (3N-6) $\sigma$ branches optiques correspondant aux vibrations internes, $6 \sigma-3$ branches optiques aux vibrations de réseau et bien entendu 3 branches acoustiques. Les courbes de dispersion correspondant à cet objet sont représentées schématiquement sur la figure 1.

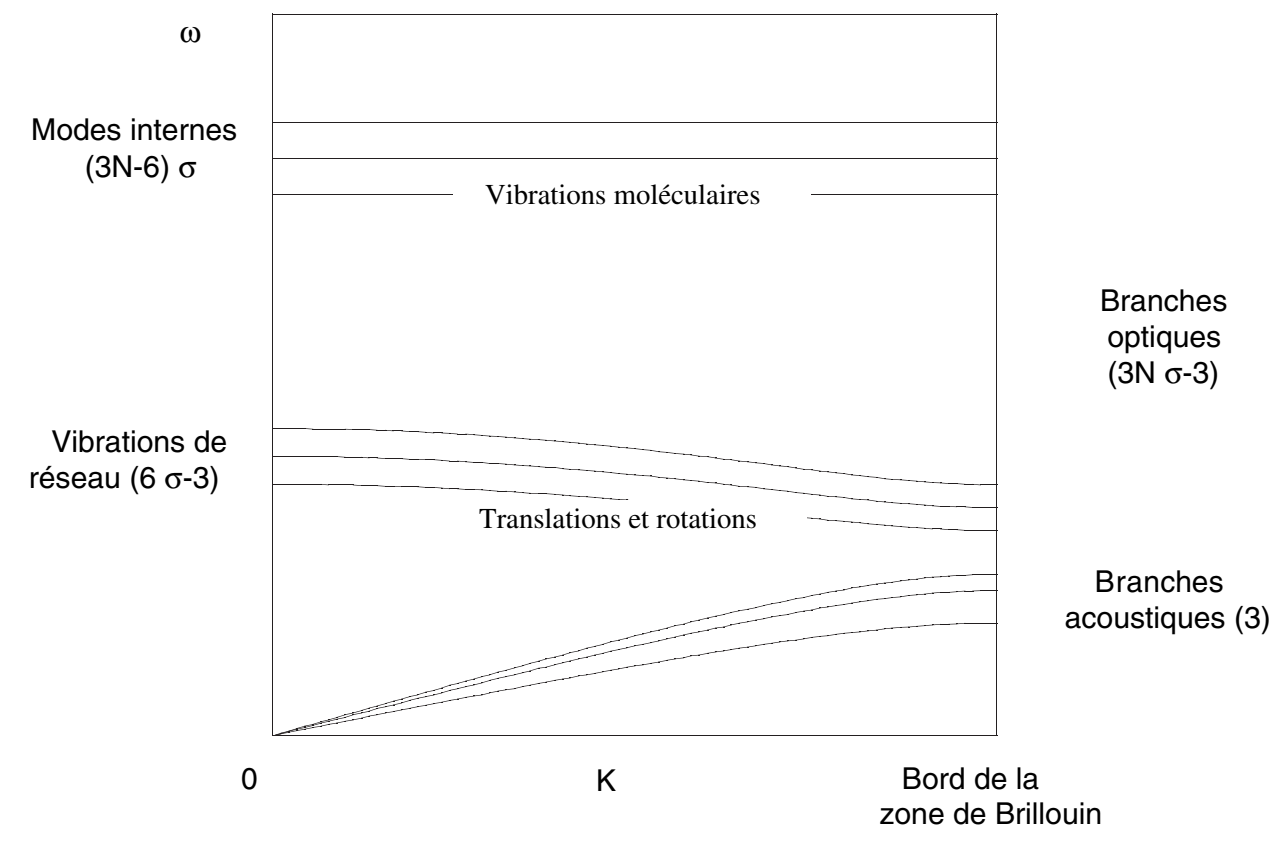

Figure 1. Représentation schématique des branches de phonons d'un cristal moléculaire.

\subsection{Le spectre électromagnétique}

Les énergies mises en jeu au cours de ces processus dynamiques sont relativement faibles, entre $1 \mu \mathrm{eV}$ et $100 \mathrm{meV}$ pour les modes externes et jusqu'à environ $500 \mathrm{meV}$ pour les modes internes. Dans le spectre électromagnétique, de telles énergies correspondent aux domaines des micro-ondes et de l'infrarouge. Il en résulte que les longueurs d'onde associées sont grandes (de quelques centimètres au micromètre) et que le vecteur d'onde correspondant est pratiquement nul. En spectroscopie optique, 
on sera donc capables d'explorer les phonons en centre de zone de Brillouin uniquement, c'est-à-dire les phonons optiques correspondant aux vibrations de réseau et aux modes internes. Il est toutefois possible de mesurer les branches acoustiques au voisinage du centre de zone par des méthodes optiques à condition de disposer d'instruments à très haute résolution : c'est la diffusion Brillouin qui fait souvent appel à des méthodes interférométriques. Quel que soit le mécanisme d'interaction entre le champ électromagnétique et le milieu étudié, nous n'aurons accès qu'aux modes actifs en centre de zone de Brillouin.

\section{INTERACTION ONDE ÉLECTROMAGNÉTIQUE - MILIEU DIÉLECTRIQUE}

Nous allons considérer, par souci de simplicité, un milieu diélectrique (neutre et où toutes les charges sont liées) en interaction avec la composante électrique $\vec{E}$ du champ électromagnétique incident. Les charges q présentes dans le milieu seront donc soumises à une force $\vec{F}$ :

$$
\vec{F}=q \vec{E}
$$

Ainsi les charges positives et négatives auront tendance à se déplacer les unes par rapport aux autres dans des directions opposées. Du fait du déplacement localisé de ces charges le milieu se polarise, c'està-dire qu'un moment dipolaire est créé. On peut par exemple imaginer un noyau entouré de son nuage d'électrons. En absence de champ électrique extérieur $\vec{E}$ les barycentres des charges positives (noyau) et négatives (électrons) coïncident. En présence de $\vec{E}$, les électrons auront tendance à se déplacer dans une direction et le noyau dans l'autre de sorte que les barycentres des charges positives et négatives ne coïncident plus, le centre de gravité de l'ensemble restant bien entendu immobile, de sorte qu'un moment électrique dipolaire apparaît. La réaction d'un milieu diélectrique à la perturbation apportée par le champ électrique extérieur conduit donc à l'apparition d'une polarisation. Si la perturbation est suffisamment faible, la réponse $\vec{P}$ (polarisation) est proportionnelle à l'excitation $\vec{E}$ et le coefficient de proportionnalité est la susceptibilité linéaire $\chi$ du milieu :

$$
\vec{P}=\chi \vec{E}
$$

En toute généralité la susceptibilité prend la forme d'un tenseur de rang deux :

$$
\vec{P}=\overleftrightarrow{\chi} \vec{E}
$$

La susceptibilité est une propriété intrinsèque du milieu considéré à l'équilibre thermodynamique et en l'absence de toute perturbation extérieure ! Ce principe est important, toute mesure par spectroscopie consiste à évaluer cette susceptibilité en perturbant légèrement le système pour en déduire ses propriétés structurales et dynamiques à l'équilibre (c'est le théorème de fluctuation-dissipation).

\subsection{Les mécanismes de polarisation d'une molécule}

Nous allons maintenant considérer une molécule simple (par exemple l'eau $\mathrm{H}_{2} \mathrm{O}$ ) en interaction avec le champ électrique $\vec{E}$. Dans un premier temps nous allons supposer que ce champ est «statique», c'est-à-dire qu'à tout instant le vecteur $\vec{E}$ est constant en norme et en direction. Très grossièrement cette molécule d'eau est constituée de 3 noyaux porteurs de charges positives et entourés d'électrons. Même en absence de champ extérieur les barycentres des charges positives et négatives ne coïncident pas pour cette molécule, elle possède un moment dipolaire permanent que l'on notera $\vec{\mu}_{0}$. C'est un vecteur qui pointe du barycentre des charges négatives $-2 \delta$ vers le barycentre des charges positives $+2 \delta$ (séparés d'une longueur 1 ) et dont la norme vaut $2 \delta 1$. Pour la molécule d'eau dans sa géométrie d'équilibre le vecteur moment dipolaire est orienté (par convention) de l'atome d'oxygène portant une charge partielle négative $-2 \delta$ vers le barycentre des charges partielles $+\delta$ portées par chacun des deux atomes d'hydrogène, comme précisé sur la figure 2. 
Lorsque cette molécule d'eau est placée dans un champ électrique externe, elle est soumise à un couple de forces ayant pour origines les barycentres des charges partielles positives et négatives. La conséquence évidente est que la molécule d'eau tendra à se réorienter de manière à aligner son moment dipolaire par rapport au champ externe. Si on imagine une assemblée isotrope de telles molécules, on voit bien que l'application d'un champ extérieur aura pour conséquence de provoquer la réorientation de tous les dipôles avec l'apparition d'un moment électrique «statistique » résultant lié à l'anisotropie ainsi crée : le milieu se polarise du fait des réorientations moléculaires avec :

$$
\vec{P}_{o r}=\chi_{o r} \vec{E}
$$

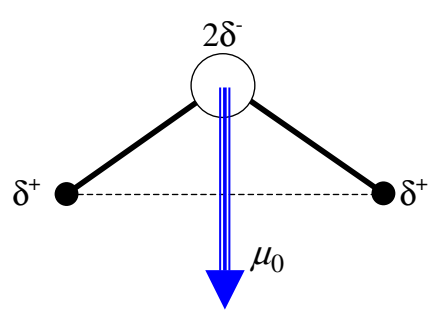

Figure 2. Représentation schématique de la molécule d'eau dans sa géométrie d'équilibre. La flèche représente le moment dipolaire permanent.

Les molécules d'eau ne sont cependant pas des entités rigides. Ainsi les porteurs de charges partielles subissant l'action du champ extérieur vont s'écarter très légèrement de la position d'équilibre ; la molécule va se déformer. Les barycentres des charges positives et négatives vont alors se déplacer très légèrement de sorte que le moment dipolaire de la molécule ne sera plus le même qu'à l'équilibre. Cette légère variation du moment dipolaire de la molécule provoquée par le champ extérieur conduira à une polarisation dite atomique ou ionique résultant de la distorsion de la molécule :

$$
\vec{P}_{a t}=\chi_{a t} \vec{E}
$$

Finalement nous n'avons considéré jusqu'ici que les porteurs de charges partielles (noyaux) pour rendre compte de la polarisation. Le nuage électronique se déforme également de sorte que si l'on imaginait une molécule indéformable, c'est-à-dire que les positions relatives des noyaux ne peuvent pas changer, on conçoit facilement que les électrons peuvent se déplacer avec l'apparition d'un moment dipolaire donnant naissance à une polarisation électronique :

$$
\vec{P}_{e l}=\chi_{e l} \vec{E}
$$

En conclusion, un milieu diélectrique soumis à un champ électrique externe statique se polarisera. La polarisation du milieu résultera à la fois des réorientations des molécules (polarisation d'orientation), de leurs déformations (polarisation atomique ou ionique) et de la déformation du nuage électronique (polarisation électronique) :

$$
\vec{P}=\left(\chi_{o r}+\chi_{a t}+\chi_{e l}\right) \vec{E}
$$

Nous allons voir par la suite que ces trois mécanismes de polarisation seront plus ou moins effectifs selon la fréquence du champ électrique oscillant.

\subsection{Mécanismes de polarisation en présence d'un champ électrique oscillant}

Nous allons maintenant considérer un champ électrique externe oscillant pour lequel, à tout instant $\mathrm{t}$, l'orientation et l'amplitude du champ électrique $\vec{E}=\vec{E}_{0} e^{-i 2 \pi v t}$ vont varier et les charges liées présentes dans le milieu vont subir les effets de ces variations selon les mécanismes énumérés au paragraphe 
précédent. Nous allons cependant considérer deux phénomènes associés à cette interaction, le premier est le phénomène de résonance (absorption) entre le diélectrique et le champ oscillant et le second est le phénomène de rayonnement d'une onde électronique lié aux déplacements périodiques des électrons par rapport aux noyaux.

\subsubsection{Le phénomène de résonance}

Le modèle le plus simple à considérer pour illustrer le phénomène de résonance est celui de la masselotte de masse $\mathrm{m}$ suspendue à un ressort et soumise à une force oscillant à la fréquence $v$. Ce modèle simpliste peut représenter une charge liée (par le ressort) soumise à la force engendrée par le champ électrique externe oscillant. Pour rendre le modèle un peu plus réaliste on supposera également que la masselotte est plongée dans un liquide de manière à apporter une friction permettant de dissiper l'énergie emmagasinée par le système vers l'extérieur (c'est le bain thermique). Le ressort est caractérisé par sa constante de raideur $\mathrm{k}$ et il est bien connu que si on écarte la masselotte de sa position d'équilibre, la masselotte se mettra à osciller périodiquement (au bout d'un certain temps) avec une fréquence caractéristique $v_{0}$ telle que :

$$
v_{0}=\frac{1}{2 \pi} \sqrt{\frac{k}{m}}
$$

Maintenant on applique une force externe telle que $\vec{F}=\vec{F}_{0} \cos (2 \pi v t)$. Si la fréquence $v$ est très inférieure à $v_{0}$, la masselotte aura tout le temps de suivre sans retard ces variations; ses oscillations et celles de la force sont en phase. Lorsque $v$ augmente un certain retard des oscillations de la masselotte par rapport à celles de la force apparaitra, en raison de son inertie. Dans le même temps les oscillations de la masselotte prendront de l'ampleur jusqu'à devenir très importantes à leur maximum lorsque $v=v_{0}$. Les phases des oscillations de la masselotte et de la force sont alors en quadrature. La friction est ici importante pour que le système n'explose pas en raison d'une trop forte accumulation d'énergie ! Lorsque la fréquence $v$ augmente encore, les oscillations de la force deviennent trop rapides pour que la masselotte, du fait de son inertie, puisse les suivre et le système ne répond plus à la perturbation. En terme d'énergie absorbée, il est bien évident qu'elle est maximale lorsque $v=v_{0}$, c'est-à-dire à la fréquence de résonance.

On peut faire maintenant un parallèle hardi avec le milieu diélectrique constitué de charges liées. Par exemple une molécule (ou un cristal atomique) peut être assimilée en première approximation à un ensemble de masselottes liées les unes aux autres par des ressorts. Un phénomène de résonance semblable à celui décrit ci-dessus se produira lorsque la fréquence de la force périodique appliquée sera égale à une des fréquences de vibration propres $v_{i}$ caractéristiques de cet ensemble d'atomes (avec $\mathrm{i}$ allant de 1 à $3 \mathrm{~N}-6$ ). Une autre condition est nécessaire pour que l'on observe une résonance : le moment dipolaire induit par un mode de vibration de la molécule doit être non nul pour qu'un couplage puisse se réaliser. Dans le milieu diélectrique, la polarisation induite s'écrira de manière similaire à l'équation (7) :

$$
\vec{P}(t)=\left(\chi_{o r}(v)+\chi_{a t}(v)+\chi_{e l}(v)\right) \vec{E}(t)
$$

Dans cette équation, la susceptibilité est maintenant une fonction complexe telle que :

$$
\chi(v)=\chi^{\prime}(v)+i \chi^{\prime \prime}(v)
$$

où la partie réelle représente l'indice de réfraction et la partie imaginaire le coefficient d'absorption. Les variations des parties réelles et imaginaires de la susceptibilité d'un diélectrique sont représentées schématiquement sur la figure 3. Les trois mécanismes de polarisation décrits sur cette figure représentent trois techniques spectroscopiques classiques bien connues, la spectroscopie des diélectriques, la spectroscopie d'absorption infrarouge et la spectroscopie d'absorption électronique 
UV-visible. Il est cependant intéressant de mentionner que dans le visible, il ne peut y avoir de phénomène de résonance lié aux vibrations moléculaires. A de telles fréquences d'excitation, seuls les électrons sont assez légers pour répondre aux sollicitations du champ électrique externe. Nous verrons ci-dessous que c'est dans cette zone de transparence que la spectroscopie de diffusion Raman est la plus utilisée, et l'on peut bien entendu en déduire que le mécanisme à l'origine de la diffusion de la lumière est lié aux électrons, donc tout à fait différent de celui correspondant à la spectroscopie infrarouge.

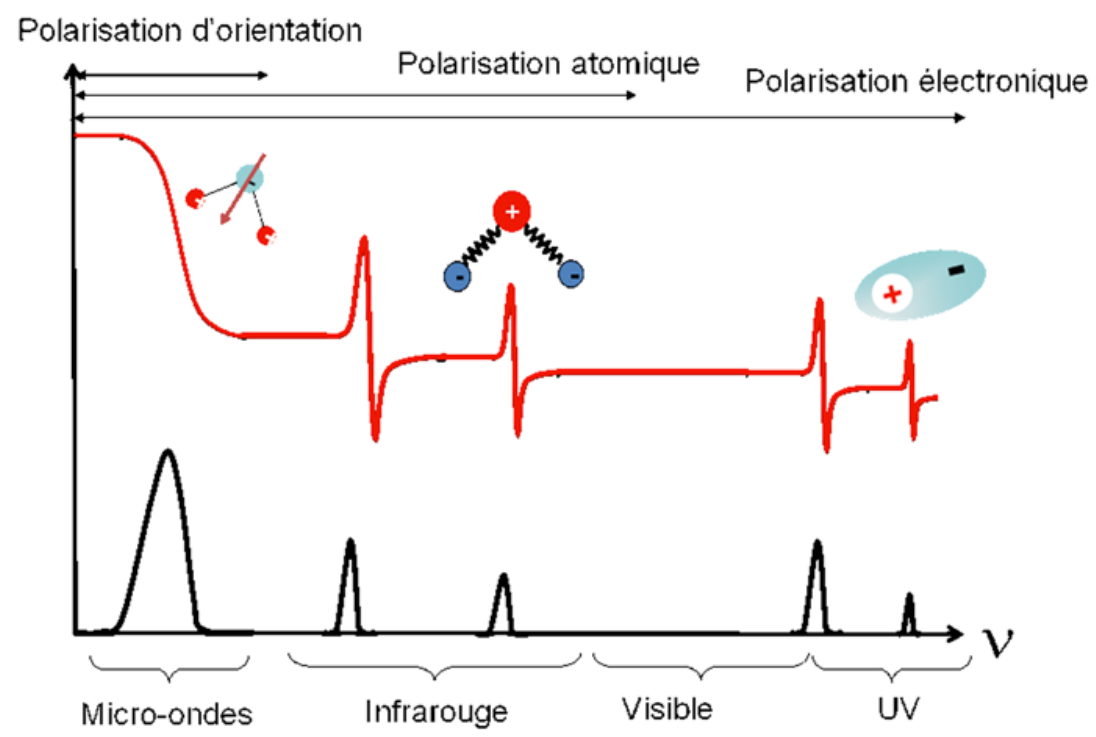

Figure 3. Représentation schématique des variations des parties réelle (courbe du haut) et imaginaire (courbe du bas) de la susceptibilité.

\subsubsection{Le dipôle oscillant}

Nous allons maintenant aborder un autre phénomène, lié à la polarisation, qui est celui de la génération d'un champ électromagnétique créé par un dipôle oscillant. Prenons par exemple le cas d'un atome constitué du noyau chargé positivement et de son nuage d'électrons. En l'absence de toute perturbation extérieure, les barycentres des charges positives et négatives coïncident. Appliquons maintenant à cet atome un champ électrique de sorte que le nuage d'électrons se déforme. Il en résultera que les barycentres des charges négatives et positives ne coïncideront plus et un moment dipolaire sera créé. Si le champ électrique appliqué oscille à une fréquence bien plus faible que toute fréquence de résonnance électronique, alors les barycentres des charges positives et négatives vont suivre ces oscillations en phase (cf. ci-dessus les oscillations forcées à des fréquences bien inférieures à la fréquence de résonance). On se retrouve dans le cas bien connu du dipôle électrique oscillant qui deviendra lui-même source d'un rayonnement électromagnétique de même fréquence que celle du rayonnement perturbateur. L'onde plane se propageant dans le plan perpendiculaire à l'axe du dipôle est polarisée parallèlement à son axe et son amplitude est nulle le long de l'axe du dipôle. Tout atome soumis à une excitation lumineuse dans le visible va réémettre un rayonnement polarisé de même fréquence que le rayonnement excitateur ; c'est la diffusion Rayleigh. L'intensité du rayonnement diffusé est inversement proportionnelle à $\lambda^{4}$. Si on considère maintenant une molécule, la situation est plus complexe. Soumise au rayonnement incident, un dipôle sera induit selon le mécanisme décrit ci-dessus et on observe une diffusion Rayleigh. Ce dipôle verra cependant ses oscillations modulées par les vibrations propres des molécules à condition que celles-ci induisent une variation de la polarisation électronique. Il en résulte qu'une diffusion inélastique 
de la lumière apparaitra à des longueurs d'ondes différentes de celle du rayonnement incident ; c'est la diffusion Raman.

\section{LES SPECTROSCOPIES DE VIBRATION INFRAROUGE ET RAMAN}

Nous avons vu dans les paragraphes précédents que les analyses de l'absorption d'un rayonnement dans l'infrarouge et de la diffusion de la lumière permettaient d'obtenir des informations sur les vibrations moléculaires et cristallines en centre de zone de Brillouin. Les mécanismes d'interaction matièrerayonnement sont très différents pour ces deux techniques et une des conséquences les plus directes de ces différences se traduit par les règles de sélection.

En effet, toutes les vibrations d'une molécule ne pourront être observées selon la technique considérée. Pour un mécanisme d'absorption du rayonnement, nous avons vu que le champ électrique externe ne peut entrer en résonance qu'avec un mode de vibration qui lui-même engendre un moment dipolaire pouvant se coupler à l'excitation. En d'autres termes la polarisation atomique s'écrira :

$$
\vec{\mu}=\vec{\mu}_{0}+\sum_{i=1}^{3 N-6}\left(\frac{\partial \vec{\mu}}{\partial q_{i}}\right)_{0} . q_{i}
$$

où les termes $\left(\frac{\partial \vec{\mu}}{\partial q_{i}}\right)_{0} . q_{i}$ représentent la variation du vecteur moment dipolaire induite par la coordonnée normale $q_{i}$ associée à chaque vibration i. De toute évidence si $\vec{\mu}_{0}$ est nul, nous n'observerons pas d'absorption dans le domaine des micro-ondes et si $\left(\frac{\partial \vec{\mu}}{\partial q_{i}}\right)_{0} . q_{i}$ est nul, le mode de vibration n'engendrera pas d'absorption dans l'infrarouge.

Similairement pour le dipôle oscillant, une vibration pourra moduler la polarisation électronique si elle engendre une variation de la polarisabilité. En d'autres termes la polarisation électronique s'écrira :

$$
\overleftrightarrow{\alpha}=\overleftrightarrow{\alpha}_{0}+\sum_{i=1}^{3 N-6}\left(\frac{\partial \overleftrightarrow{\alpha}}{\partial q_{i}}\right)_{0} \cdot q_{i}
$$

où $\overleftrightarrow{\alpha}_{0}$ est le tenseur de polarisabilité «permanent» et les termes $\left(\frac{\partial \overleftrightarrow{\alpha}}{\partial q_{i}}\right)_{0} . q_{i}$ représentent la variation du tenseur de polarisabilité induite par chaque vibration i. De toute évidence $\overleftrightarrow{\alpha}_{0}$ n'est jamais nul (il y aura toujours de la diffusion Rayleigh) et $\left(\frac{\partial \overleftrightarrow{\alpha}}{\partial q_{i}}\right)_{0} . q_{i}$ doit être différent de zéro pour conduire à une diffusion Raman. On peut se rendre compte, à travers l'exemple de la molécule d'eau, que la géométrie du milieu (molécule, cristal) et donc les propriétés de symétrie du système vont jouer un rôle déterminant dans ces mécanismes d'absorption et de diffusion.

\subsection{Dénombrement et règles de sélection}

Une manière élégante et efficace de dénombrer les vibrations d'une molécule ou d'un cristal et de préciser les règles de sélection qui y sont associées est de tirer profit des propriétés de symétrie du système dans son état d'équilibre à l'aide des principes de base de la théorie des groupes. Grâce à une telle analyse, sans effectuer le moindre calcul, on peut déterminer le nombre et les propriétés de symétrie des vibrations moléculaires ainsi que leur activité en infrarouge et Raman. Dans le cadre de ce cours de sensibilisation aux spectroscopies optiques, nous allons nous contenter d'introduire ces concepts issus de la théorie des groupes et de les illustrer par quelques exemples simples. 


\subsubsection{Dénombrement}

Chaque molécule peut être décrite par son groupe de symétrie ou groupe ponctuel. Chaque représentation irréductible peut être spécifiée par ses caractères que l'on rassemble dans un tableau. Ce tableau de caractères va nous permettre de dénombrer les différents degrés de liberté et de leur associer les classes de symétrie correspondantes. Les différents éléments constitutifs de la table des caractères sont définis sur la figure 4.

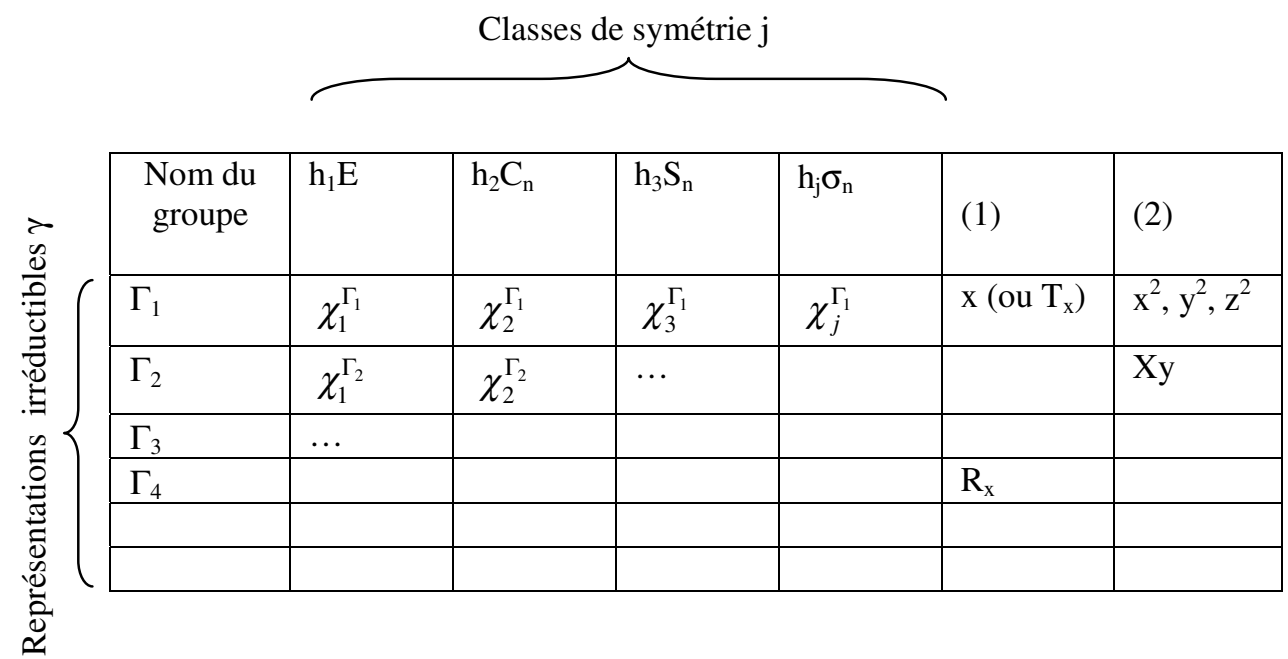

Figure 4. Table des caractères $\chi_{j}$ et ses éléments : (1) Représentations engendrées par les coordonnées d'un vecteur : (2) Représentations engendrées par les coordonnées d'un tenseur.

Pour réaliser ce dénombrement on appliquera la relation suivante :

$$
n^{(\gamma)}=\frac{1}{h} \sum_{R} h_{j} \chi_{j}^{(\gamma) *} m_{j}\left(2 \cos \phi_{j} \pm 1\right)
$$

où $h_{\mathrm{j}}$ est le nombre d'opérations de symétrie $\mathrm{R}$ appartenant à la classe $\mathrm{j}, \chi_{j}^{(\Gamma)}$ le caractère de la représentation $\Gamma$ d'une opération appartenant à la classe $\mathrm{j}$. L'ordre du groupe $h=\sum_{\mathrm{R}} h_{j}$ et $\mathrm{m}_{\mathrm{j}}$ est le nombre d'atomes restant fixes ou qui sont transportés en des atomes congruents selon une opération de symétrie appartenant à la classe $\mathrm{j}$. Les Tout groupe termes $2 \cos \phi_{j} \pm 1$ sont reportés dans la table 1 pour les différentes opérations de symétrie.

Tableau 1. Termes $2 \cos \phi_{j} \pm 1$ pour les différentes opérations de symétrie.

\begin{tabular}{|l|c|c|c|c|c|}
\hline Opérations de symétrie & $\mathrm{E}$ & $\mathrm{C}_{n}$ & $\mathrm{~S}_{n}$ & $\mathrm{i}$ & $\sigma$ \\
\hline $2 \cos \phi_{j} \pm 1$ & 3 & $2 \cos \frac{2 \pi}{n}+1$ & $2 \cos \frac{2 \pi}{n}-1$ & -3 & 1 \\
\hline
\end{tabular}

\subsubsection{Règles de sélection}

Reprenons l'exemple de l'eau déjà abordé auparavant. Dans son état d'équilibre, les éléments de symétrie de la molécule d'eau (figure 5) sont : un axe de symétrie d'ordre deux $\mathrm{C}_{2}$ définissant l'axe $\mathrm{z}$ du repère et deux plans de symétrie (ou plans miroirs) $\sigma_{\mathrm{v}}(\mathrm{xz})$ et $\sigma_{\mathrm{v}}^{\prime}(\mathrm{yz})$. Tout groupe ponctuel contient une représentation irréductible invariante selon toutes les opérations de symétrie du groupe et dont les caractères sont tous égaux à 1 . C'est la représentation totalement symétrique $A_{1}$ du groupe $C_{2 v}$ (voir 
table 2). Appliquons maintenant la formule (13) pour dénombrer les degrés de liberté de la molécule d'eau. Nous aurons :

$$
\begin{aligned}
& n^{\left(A_{1}\right)}=\frac{1}{4}[(1 \times 1 \times 3 \times 3)+(1 \times 1 \times 1 \times(-1))+(1 \times 1 \times 3 \times 1)+(1 \times 1 \times 1 \times 1)]=3 \\
& n^{\left(A_{2}\right)}=\frac{1}{4}[(1 \times 1 \times 3 \times 3)+(1 \times 1 \times 1 \times(-1))+(1 \times(-1) \times 3 \times 1)+(1 \times(-1) \times 1 \times 1)]=1 \\
& n^{\left(B_{1}\right)}=\frac{1}{4}[(1 \times 1 \times 3 \times 3)+(1 \times(-1) \times 1 \times(-1))+(1 \times 1 \times 3 \times 1)+(1 \times(-1) \times 1 \times 1)]=3 \\
& n^{\left(B_{2}\right)}=\frac{1}{4}[(1 \times 1 \times 3 \times 3)+(1 \times(-1) \times 1 \times(-1))+(1 \times(-1) \times 3 \times 1)+(1 \times 1 \times 1 \times 1)]=2
\end{aligned}
$$

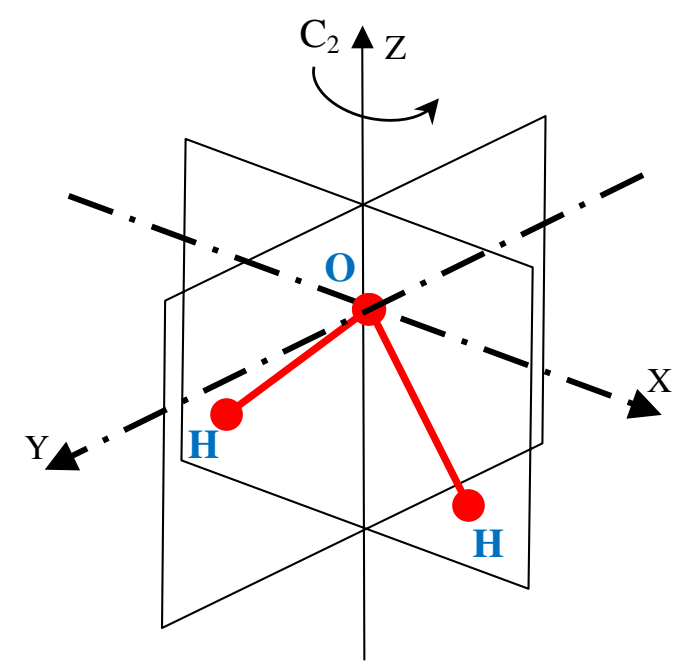

Figure 5. Les éléments de symétrie du groupe $C_{2 v}$ de la molécule d'eau.

Soient les 9 degrés de liberté attendus qui se déclinent selon :

$$
\Gamma_{\text {total }}=3 A_{1}+A_{2}+3 B_{1}+2 B_{2}
$$

La lecture de la table des caractères (table 2) nous permet d'identifier les translations de symétries $\mathrm{A}_{1}$, $\mathrm{B}_{1}$ et $\mathrm{B}_{2}$ de même que les rotations $\mathrm{A}_{2}, \mathrm{~B}_{1}$ et $\mathrm{B}_{2}$. Nous aurons donc, après avoir retranché ces degrés de liberté de la formule (14), les représentations associées aux 3 modes de vibration :

$$
\Gamma_{v i b}=2 A_{1}+B_{1}
$$

Tableau 2. La table des caractères du groupe $\mathrm{C}_{2 \mathrm{v}}$.

\begin{tabular}{|c|cccc|c|c|}
\hline $\mathrm{C}_{2 \mathrm{v}}$ & $\mathrm{E}$ & $\mathrm{C}_{2}$ & $\sigma_{\mathrm{v}}(\mathrm{xz})$ & $\sigma^{\prime}{ }_{\mathrm{v}}(\mathrm{yz})$ & & \\
\hline $\mathrm{A}_{1}$ & 1 & 1 & 1 & 1 & $\mathrm{z}$ & $\mathrm{x}^{2} ; \mathrm{y}^{2} ; \mathrm{z}^{2}$ \\
$\mathrm{~A}_{2}$ & 1 & 1 & -1 & -1 & $\mathrm{R}_{\mathrm{z}}$ & $\mathrm{xy}$ \\
$\mathrm{B}_{1}$ & 1 & -1 & 1 & -1 & $\mathrm{x} ; \mathrm{R}_{\mathrm{y}}$ & $\mathrm{xz}$ \\
$\mathrm{B}_{2}$ & 1 & -1 & -1 & 1 & $\mathrm{y} ; \mathrm{R}_{\mathrm{x}}$ & $\mathrm{yz}$ \\
\hline
\end{tabular}

Examinons maintenant les mouvements possibles des atomes pour les vibrations de l'eau. On attend 3 modes de vibration correspondant à des élongations des liaisons $\mathrm{O}-\mathrm{H}$ ou des variations d'angle $H \hat{O} H$ schématisées sur la figure 6. Si on considère des élongations en phase des liaisons $\mathrm{O}-\mathrm{H}$ (mode que l'on notera $v_{\mathrm{s}}$ ), il est bien évident que cette vibration est symétrique par rapport à toutes les opérations du groupe ponctuel (la molécule $\mathrm{H}_{2} \mathrm{O}$ conserve sa symétrie $\mathrm{C}_{2 \mathrm{v}}$ au cours de cette vibration) de sorte que ce mode appartient à la représentation totalement symétrique $\mathrm{A}_{1}$. Il en est de même pour la vibration 
mettant en jeu l'angle $H \hat{O} H$ (que l'on notera $\delta$ ). En revanche, si on considère la vibration mettant en jeu des élongations des liaisons $\mathrm{O}-\mathrm{H}$ en opposition de phase, les opérations de symétrie $\mathrm{C}_{2}$ et $\sigma_{\mathrm{v}}^{\prime}$ n'existent plus dans la molécule déformée en cour de vibration et le caractère associé aux opérations $\mathrm{C}_{2}$ et $\sigma_{v}^{\prime}$ sera -1 . Ce mode, que l'on notera $v_{\text {as }}$, appartient à la représentation antisymétrique $\mathrm{B}_{1}$. Au total, les trois modes de vibration de la molécule d'eau appartiendront aux représentations irréductibles $\mathrm{A}_{1}$ (pour les vibrations $v_{\mathrm{s}}$ et $\delta$ ) et $\mathrm{B}_{1}$ (pour la vibration $v_{\mathrm{as}}$ ) du groupe ponctuel $\mathrm{C}_{2 \mathrm{v}}$.

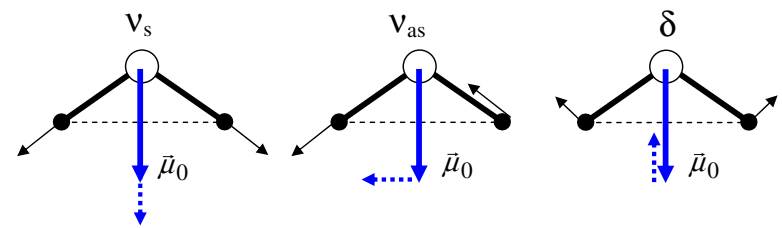

Figure 6. Représentation schématique des 3 modes de vibration internes de la molécule d'eau. Les déplacements des atomes sont symbolisés par une flèche en traits pleins et le moment dipolaire induit $\partial \vec{\mu} \partial \vec{\mu} \partial q \partial q$ est symbolisé par la flèche en pointillés.

La table des caractères regroupe un ensemble d'informations capitales en spectroscopie, en particulier les règles de sélection et des propriétés géométriques. Nous avons vu qu'une condition nécessaire pour qu'un mode de vibration soit actif en infrarouge est que la vibration provoquant la déformation de la molécule induise un moment dipolaire. Comme schématisé sur la figure 6 , le moment dipolaire induit par les vibrations totalement symétriques $v_{\mathrm{s}}$ et $\delta$ est colinéaire à l'axe z de la molécule et ces vibrations sont donc actives en infrarouge. Ces informations sur l'activité des modes ainsi que les orientations des moments dipolaires induits sont données par la table des caractères. Nous voyons (table 2) que les modes de symétrie $A_{1}$ sont actifs en infrarouge car ils se transforment selon la représentation du vecteur translation $\mathrm{z}$. Le mode $\mathrm{B}_{1}$ est aussi actif en infrarouge car il se transforme selon la représentation du vecteur translation $\mathrm{x}$, indiquant aussi que le moment dipolaire induit par la vibration est colinéaire à l'axe x de la molécule (figure 6). Pour la diffusion Raman, la variable de couplage est le tenseur de polarisabilité. L'activité Raman sera donc, par analogie, liée aux variations de la géométrie (périgée et/ou apogée) et/ou de la direction du grand axe du tenseur de polarisabilité. Ces informations sont également fournies dans les tables de caractères; les vibrations actives en Raman appartiennent à des représentations irréductibles selon lesquelles se transforment les différentes composantes d'un tenseur. Ainsi pour la molécule d'eau (table 2), les trois modes de vibration seront également actifs en Raman, les tenseurs induits pour modes $\mathrm{A}_{1}$ et $\mathrm{B}_{1}$ auront les formes :

$$
A_{1}:\left(\frac{\partial \overleftrightarrow{\alpha}}{\partial q}\right)=\left[\begin{array}{lll}
a & 0 & 0 \\
0 & b & 0 \\
0 & 0 & c
\end{array}\right] \quad B_{1} \quad: \quad\left(\frac{\partial \overleftrightarrow{\alpha}}{\partial q}\right)=\left[\begin{array}{lll}
0 & 0 & d \\
0 & 0 & 0 \\
d & 0 & 0
\end{array}\right]
$$

Nous venons de voir que toutes les vibrations de l'eau, qui est une molécule polaire, sont actives en infrarouge et en Raman. Qu'en est-il pour une molécule apolaire ? Nous allons maintenant examiner le cas de la molécule d'éthylène qui est de symétrie $\mathrm{D}_{2 \mathrm{~h}}$ (figure 7). Cette molécule possède un centre d'inversion et ne présente donc pas de moment dipolaire permanent. Ainsi les modes de vibration totalement symétriques $A_{g}$ ne pourront pas être actifs en infrarouge (cf. table 3 ). Il en sera de même pour tous les modes de vibration symétriques par rapport au centre d'inversion, c'est-à-dire pour toutes les représentations pour lesquelles le caractère associé à l'opération inversion (i) est $1\left(\mathrm{~B}_{1 \mathrm{~g}}, \mathrm{~B}_{2 \mathrm{~g}}\right.$ et $\mathrm{B}_{3 \mathrm{~g}}$ ). Lorsqu'on examine plus attentivement la table des caractères du groupe, on s'aperçoit que les modes de vibration non symétriques par rapport au centre d'inversion (caractère -1) seront actifs en infrarouge, à l'exception du mode $\mathrm{A}_{\mathrm{u}}$. Le dénombrement (équation (13)) des degrés de liberté de la 
molécule d'éthylène donne :

$$
\Gamma_{\text {total }}=3 A_{g}+3 B_{1 g}+B_{2 g}+2 B_{3 g}+A_{u}+2 B_{1 u}+3 B_{2 u}+3 B_{3 u}
$$

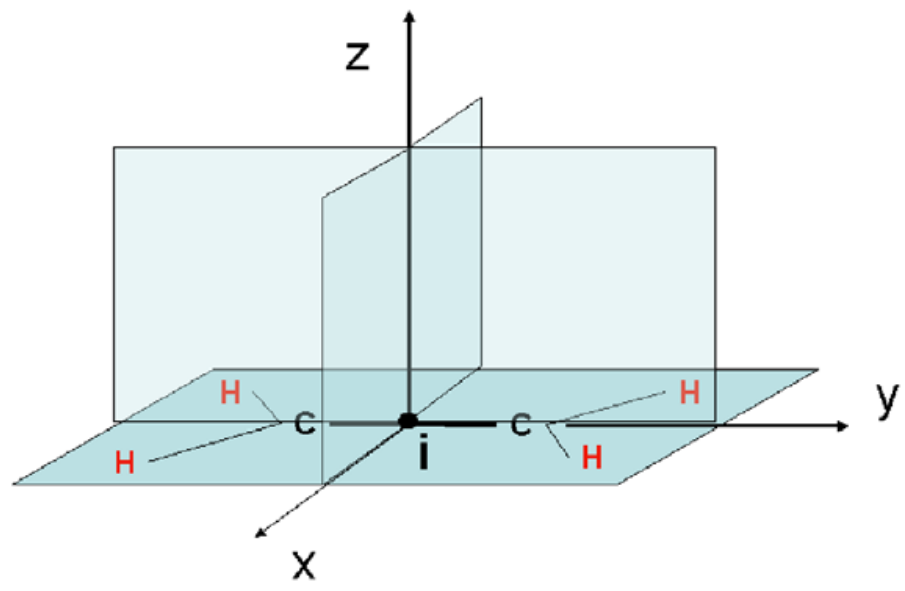

Figure 7. Eléments de symétrie de la molécule d'éthylène.

Et le dénombrement des vibrations actives en infrarouge donne, après avoir retranché les translations :

$$
\Gamma_{I R}=B_{1 u}+2 B_{2 u}+2 B_{3 u}
$$

A partir de la table des caractères nous pouvons déduire, pour chaque mode de vibration de la molécule d'éthylène, par rapport à quel axe le moment dipolaire induit est colinéaire. Par exemple le mode de vibration des élongations des liaisons $\mathrm{C}-\mathrm{H}$ schématisé sur la figure 8 appartenant à la représentation $\mathrm{B}_{2 u}$ induira un moment dipolaire colinéaire à l'axe y de la molécule et la vibration $\mathrm{B}_{3 u}$ schématisée sur la figure 8 un moment dipolaire colinéaire à l'axe x. Toutes ces informations sont rassemblées dans la table des caractères (table 3 ). Le dénombrement des vibrations actives en Raman donne, après avoir retranché les rotations :

$$
\Gamma_{R}=3 A_{g}+2 B_{1 g}+B_{3 g}
$$

Tableau 3. La table des caractères du groupe $\mathrm{D}_{2 \mathrm{~h}}$.

\begin{tabular}{|c|cccccccc|c|c|}
\hline $\mathrm{D}_{2 \mathrm{~h}}$ & $\mathrm{E}$ & $\mathrm{C}_{2}(\mathrm{z})$ & $\mathrm{C}_{2}(\mathrm{y})$ & $\mathrm{C}_{2}(\mathrm{x})$ & $\mathrm{i}$ & $\sigma(\mathrm{xy})$ & $\sigma(\mathrm{xz})$ & $\sigma(\mathrm{yz})$ & & \\
\hline $\mathrm{A}_{g}$ & 1 & 1 & 1 & 1 & 1 & 1 & 1 & 1 & & $\mathrm{x}^{2} ; \mathrm{y}^{2} ; \mathrm{z}^{2}$ \\
$\mathrm{~B}_{1 \mathrm{~g}}$ & 1 & 1 & -1 & -1 & 1 & 1 & -1 & -1 & $\mathrm{R}_{\mathrm{z}}$ & $\mathrm{xy}$ \\
$\mathrm{B}_{2 \mathrm{~g}}$ & 1 & -1 & 1 & -1 & 1 & -1 & 1 & -1 & $\mathrm{R}_{\mathrm{y}}$ & $\mathrm{xz}$ \\
$\mathrm{B}_{3 \mathrm{~g}}$ & 1 & -1 & -1 & 1 & 1 & -1 & -1 & 1 & $\mathrm{R}_{\mathrm{x}}$ & $\mathrm{yz}$ \\
$\mathrm{A}_{\mathrm{u}}$ & 1 & 1 & 1 & 1 & -1 & -1 & -1 & -1 & & \\
$\mathrm{~B}_{1 \mathrm{u}}$ & 1 & 1 & -1 & -1 & -1 & -1 & 1 & 1 & $\mathrm{z}$ & \\
$\mathrm{B}_{2 u}$ & 1 & -1 & 1 & -1 & -1 & 1 & -1 & 1 & $\mathrm{y}$ & \\
$\mathrm{B}_{3 u}$ & 1 & -1 & -1 & 1 & -1 & 1 & 1 & -1 & $\mathrm{x}$ & \\
\hline
\end{tabular}

Nous avons vu ci-dessus que les règles de sélection en absorption infrarouge étaient liées aux variations du moment dipolaire induit par les vibrations de la molécule. Ainsi, pour l'exemple de la molécule d'éthylène présenté ci-dessus, nous voyons que seuls les modes de vibration symétriques par rapport au centre d'inversion seront actifs en Raman. Il est intéressant de mentionner la «règle 

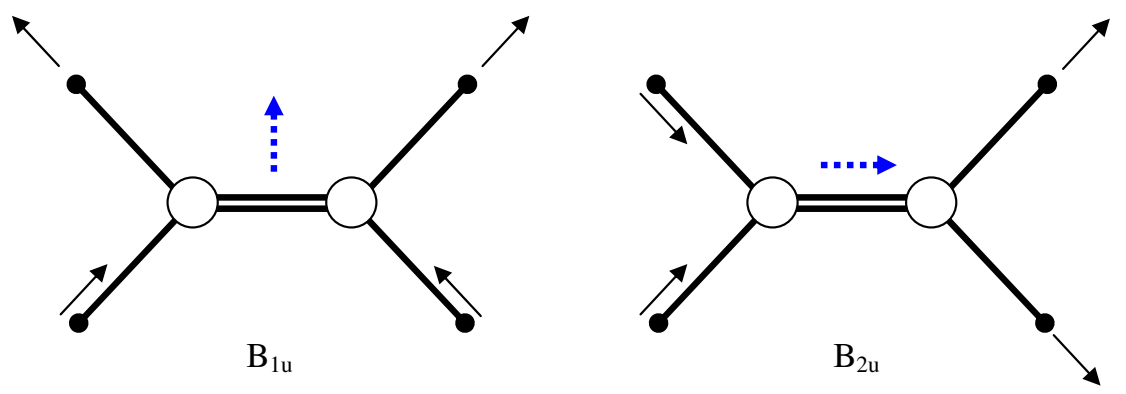

Figure 8. Représentation schématique des modes de vibration des élongations des liaisons $\mathrm{C}-\mathrm{H}$ appartenant aux représentations $\mathrm{B}_{1 u}$ et $\mathrm{B}_{2 u}$ de la molécule d'éthylène. Les déplacements des atomes sont symbolisés par une flèche en traits pleins et le moment dipolaire induit $\partial \vec{\mu} / \partial \vec{\mu} \partial q \partial q$ est symbolisé par la flèche en pointillés.

d'exclusion» qui découle de l'exemple de l'éthylène : pour un système centro-symétrique, toutes les vibrations actives en infrarouge sont inactives en Raman et vice-versa. Il est aussi intéressant de remarquer qu'il existe des modes dits «silencieux» (le mode $A_{u}$ du groupe $D_{2 h}$ ), inactifs à la fois en infrarouge et en Raman (mais pas inactifs en diffusion neutronique qui est une spectroscopie sans règles de sélections !).

\section{2 Études en lumière polarisée}

Nous définissons l'activité infrarouge d'un mode de vibration à travers le vecteur moment dipolaire induit défini dans l'équation (11). On peut facilement imaginer orienter un cristal de manière à ce que pour un mode de vibration donné le moment dipolaire induit soit perpendiculaire au vecteur champ électrique du rayonnement incident (à condition bien entendu de réaliser une expérience avec un rayonnement incident polarisé). Dans ce cas, ce mode de vibration bien qu'actif en infrarouge, sera inobservable pour des raisons purement géométriques. Ce mode pourra par contre être observé si le moment dipolaire induit est parallèle (ou possède une composante parallèle) au vecteur champ électrique du rayonnement incident. On retrouve ce même principe en diffusion neutronique avec le produit scalaire entre le vecteur transfert de moment et le vecteur déplacement de l'atome. En revanche il n'y a pas à proprement dit de règle de sélection en diffusion neutronique (c'est-à-dire que la section efficace de diffusion ne devient pas nulle pour certaines vibrations), contrairement aux spectroscopies optiques.

Nous avons mentionné ci-dessus les règles d'extinction «géométriques» pour l'absorption en présence d'un champ excitateur polarisé. En diffusion Raman des règles similaires existent mais légèrement plus complexes puisque la variable de couplage système/excitation est ici un tenseur de rang deux. Prenons pour exemple le schéma reporté sur la figure 9 représentant un système quelconque soumis à un rayonnement incident polarisé selon l'axe $\mathrm{OZ}$ du laboratoire. Pour un système de symétrie sphérique, le rayonnement diffusé dans une direction perpendiculaire à la direction du champ incident sera nécessairement polarisé selon l'axe du dipôle induit, c'est à dire OZ. Pour une molécule de symétrie quelconque l'axe du dipôle oscillant n'est plus nécessairement parallèle au champ électrique du champ incident mais peut prendre une autre direction. Il en résulte, par exemple pour une polarisation incidente parallèle à l'axe $\mathrm{OZ}$, que le vecteur champ électrique du rayonnement diffusé selon la géométrie expérimentale de la figure 9 présentera une composante non nulle selon OY. Ainsi on peut analyser indépendamment les éléments du tenseur de polarisabilité en disposant d'un champ incident de polarisation connue et en plaçant, pour l'analyse, un polariseur permettant de mesurer sélectivement la projection du champ diffusé selon un des axes du laboratoire. Ici aussi la table des caractères nous permettra, pour chaque vibration active en Raman, de déduire la forme du tenseur de polarisabilité induit par la vibration ou plus précisément le terme $\left(\frac{\partial \overleftrightarrow{\alpha}}{\partial q}\right)$ de l'équation (12). 


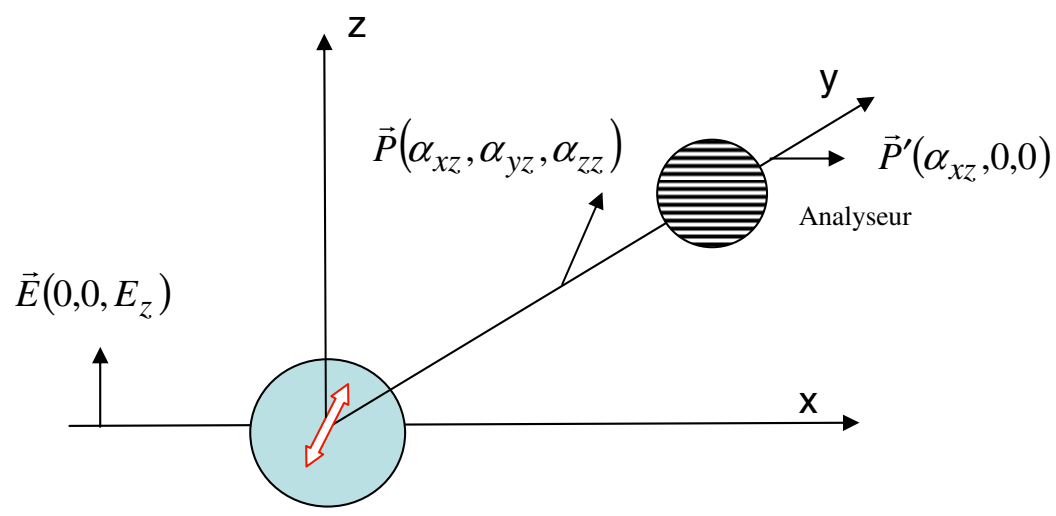

Figure 9. Représentation schématique du montage permettant de déterminer la composante xz du tenseur de polarisabilité. La double flèche représente le dipôle induit.

\section{DYNAMIQUE CRISTALLINE ET SPECTROSCOPIE OPTIQUE}

Nous allons maintenant appliquer les principes énumérés ci-dessus à l'étude de cristaux. Nous nous intéressons uniquement aux modes de centre de zone, c'est à dire correspondant à $\mathrm{K}=0$ et qui sont susceptibles d'être actifs en infrarouge et en diffusion Raman dans les processus du premier ordre (processus à un phonon). Bien entendu la théorie des groupes va jouer un rôle déterminant pour l'interprétation des spectres expérimentaux. Nous allons en premier lieu aborder le cas plus simple des cristaux atomiques avant d'aborder le cas plus complexe des cristaux moléculaires.

\subsection{Méthode du groupe facteur}

La méthode du groupe facteur consiste à considérer une seule maille (primitive) du cristal et à la traiter comme une molécule polyatomique. Cette méthode relativement directe permet de traiter les cristaux «atomiques» de type $\mathrm{NaCl}, \mathrm{NiO}$, diamant etc.

Prenons comme premier exemple le cas du cristal $\mathrm{NaCl}$. C'est un cristal cubique appartenant au groupe d'espace $F m \overline{3} m-O_{\mathrm{h}}^{5}$ du système cubique à faces centrées. Les ions $\mathrm{Cl}^{-}$occupent des sites dont la symétrie est $\left(\mathrm{O}_{\mathrm{h}}\right)$ et les ions $\mathrm{Na}^{+}$occupent des sites dont la symétrie est également $\left(\mathrm{O}_{\mathrm{h}}\right)$. Le dénombrement des vibrations doit être effectué dans la maille primitive et non dans la maille cristallographique de multiplicité 4 . Compte tenu de cette remarque, la maille primitive contient une seule unité formulaire $\mathrm{NaCl}$, et les ions $\mathrm{Cl}^{-}$et $\mathrm{Na}^{+}$restent invariants selon toutes les opérations de symétrie du groupe facteur $\mathrm{O}_{\mathrm{h}}$. Puisque la maille primitive contient 2 atomes, 6 degrés de liberté sont attendus incluant les 3 modes de translations d'ensemble (modes acoustiques). Le dénombrement des vibrations est alors très simple, la table des caractères du groupe $\mathrm{O}_{\mathrm{h}}$ montre que les 6 degrés de liberté appartiennent à la représentation triplement dégénérée $F_{1 u}$. On attend donc un seul phonon optique triplement dégénéré $\mathrm{F}_{1 \mathrm{u}}$ actif en infrarouge et aucun mode actif en Raman.

Un autre exemple intéressant est celui du diamant qui appartiennt au groupe d'espace $F d \overline{3} m-O_{\mathrm{h}}^{7}$ du système cubique à faces centrées. Il s'agit d'un cristal homo nucléaire où les atomes de carbone occupent des positions dont la symétrie de site est $\overline{4} 3 m\left(T_{d}\right)$. La maille cristallographique contient 8 atomes, mais la maille primitive n'en contient que 2, et ces atomes seront donc invariants selon les opérations qui constituent le groupe de site $\mathrm{T}_{\mathrm{d}}$. Le dénombrement est effectué en appliquant la formule (13) dans le groupe $\mathrm{O}_{h}$ et en tenant compte du fait que les facteurs $\mathrm{m}_{\mathrm{j}}$ sont non nuls uniquement pour les opérations de symétrie du groupe $\mathrm{T} 4_{\mathrm{d}}$ :

$$
\Gamma_{\text {total }}=F_{2 g}+F_{1 u}
$$


Les translations appartiennent à la représentation $F_{1 u}$ et le phonon optique triplement dégénéré $F_{2 g}$ est actif en Raman mais inactif en infrarouge. Le diamant, comme le silicium et le germanium, sont donc très intéressants pour leur transparence dans tout le domaine de l'infrarouge. On les utilise en effet comme fenêtres ou comme prismes pour des montages de réflexion totale atténuée.

\subsection{Méthode des corrélations}

Dans le paragraphe précédent, nous avons vu que la méthode du groupe facteur est très efficace et rapide dans le cas de cristaux ioniques de structures simples, mais elle devient vite d'application plus difficile dans les cristaux moléculaires pour lesquels le nombre d'atomes contenus dans la maille élémentaire peut devenir très élevé. Dans un cristal moléculaire, chaque molécule de la maille pourra être déformée par rapport à sa géométrie à l'état isolé sous l'action du champ cristallin. De plus des couplages intermoléculaires entre vibrateurs peuvent être importants. La méthode des corrélations nous permettra de dénombrer toutes les vibrations en centre de zone de Brillouin pour un cristal moléculaire.

Avec la méthode des corrélations, on part du dénombrement des vibrations de la molécule isolée. Le groupe de site étant un sous-groupe du groupe ponctuel de symétrie de la molécule isolée, on obtient aisément par corrélation (relation de groupe à sous-groupe) le dénombrement des modes de vibration de la molécule dans son site, c'est à dire lorsqu'elle est soumise à des déformations dues au champ cristallin. Le groupe de site étant lui-même un sous groupe du groupe facteur, une autre corrélation va nous conduire aux modes de vibrations des différentes molécules constituant la maille élémentaire.

Pour illustrer cette méthode nous allons prendre comme exemple le bromure d'ammonium. Le bromure d'ammonium $\mathrm{NH}_{4} \mathrm{Br}$ cristallise à température ambiante dans le système cubique selon le groupe d'espace $P m \overline{3} m-O_{\mathrm{h}}^{1}$. La maille élémentaire contient une seule unité formulaire et les ions ammonium présentent un désordre entre deux orientations énergétiquement équivalentes. Ce cristal subit une transition de phase structurale à $T_{c}=235 \mathrm{~K}$ vers une phase quadratique ordonnée où les ions ammonium sont orientés alternativement dans l'une et l'autre orientation possible, selon un ordre de type "antiferro" (on peut pour plus de détails se référer à l'article de R. Currat [2]). La maille élémentaire contient donc deux unités formulaires et le groupe d'espace est $P 4 / \mathrm{nmm}-D_{4 \mathrm{~h}}^{7}$. Les ions $\mathrm{NH}_{4}^{+}$occupent des sites de symétrie $\overline{4} 2 m-D_{2 \mathrm{~d}}$ et les ions $\mathrm{Br}^{-}$des sites $4 m m-C_{4 v}$.

L'ion $\mathrm{NH}_{4}^{+}$isolé possède la symétrie $\mathrm{T}_{\mathrm{d}}$ et le dénombrement de ses vibrations selon la formule (13) conduit à la répartition suivante (les rotations appartiennent à la représentation $\mathrm{F}_{1}$ et les translations à $\left.\mathrm{F}_{2}\right)$;

$$
\Gamma_{v i b}\left(N H_{4}^{+}\right)=A_{1}+E+2 F_{2}
$$

$\mathrm{L}^{\prime}$ ion $\mathrm{Br}^{-}$, qui occupe un site $\mathrm{C}_{4 v}$, donnera lieu à 3 degrés de liberté de représentations $\mathrm{A}_{1}+\mathrm{E}$.

Nous allons maintenant examiner les relations de compatibilité entre le groupe ponctuel $\mathrm{T}_{\mathrm{d}}$ de l'ion ammonium isolé, le groupe ponctuel $\mathrm{D}_{2 \mathrm{~d}}$ de l'ion ammonium dans son site et le groupe $\mathrm{D}_{4 \mathrm{~h}}$ de la maille élémentaire, ainsi qu'entre le groupe ponctuel $\mathrm{C}_{4 v}$ de l'ion bromure dans son site et le groupe $\mathrm{D}_{4 \mathrm{~h}}$ de la maille. Le groupe $\mathrm{D}_{2 \mathrm{~d}}$ est un sous groupe de $\mathrm{T}_{\mathrm{d}}$. L'examen des tables de caractères de ces deux groupes va nous permettre d'établir le diagramme des corrélations. Il faut pour cela ne considérer que les opérations de symétrie communes $\mathrm{E}, \mathrm{C}_{2}, \mathrm{~S}_{4}$ et $\sigma$ et trouver les combinaisons de représentations du sous-groupe $\mathrm{D}_{2 \mathrm{~d}}$ qui conduisent aux représentations de $\mathrm{T}_{\mathrm{d}}$. Nous avons reporté dans la table 4 les éléments des tables de caractères permettant d'établir le diagramme des corrélations. Par exemple les caractères de la représentation $\mathrm{A}_{1}$ de $\mathrm{T}_{\mathrm{d}}$ sont identiques à ceux de la représentation $\mathrm{A}_{1}$ de $\mathrm{D}_{2 \mathrm{~d}}$. Ceux de la représentation $A_{2}$ dans $T_{d}$ sont identiques à ceux de $B_{1}$ dans $D_{2 d}$. De même on retrouvera les caractères de la représentation $E$ dans $T_{d}$ en faisant la somme des caractères des représentations $A_{1}$ et $\mathrm{B}_{1}$ dans $\mathrm{D}_{2 \mathrm{~d}}$ et ainsi de suite. On applique la même méthode pour établir les relations de compatibilité entre $\mathrm{D}_{2 \mathrm{~d}}$ et $\mathrm{D}_{4 \mathrm{~h}}$ pour parvenir au diagramme reporté sur la figure 10. 
Tableau 4. Eléments des tables des caractères de $r m D_{2 \mathrm{~d}}$ et $\mathrm{T}_{\mathrm{d}}$ permettant d'établir les relations de compatibilité.

\begin{tabular}{|c|ccccc|l}
\hline $\mathrm{D}_{2 \mathrm{~d}}$ & $\mathrm{E}$ & $2 \mathrm{~S}_{4}$ & $\mathrm{C}_{2}$ & $2 \mathrm{C}_{2}^{\prime}$ & $\mathrm{y} \sigma_{\mathrm{d}}$ & \\
$\mathrm{A}_{1}$ & 1 & 1 & 1 & 1 & 1 & \\
$\mathrm{~A}_{2}$ & 1 & 1 & 1 & -1 & -1 & \\
$\mathrm{~B}_{1}$ & 1 & -1 & 1 & 1 & -1 & \\
$\mathrm{~B}_{2}$ & 1 & -1 & 1 & -1 & 1 & \\
$\mathrm{E}$ & 2 & 0 & -2 & 0 & 0 & \\
\hline $\mathrm{T}_{\mathrm{d}}$ & $\mathrm{E}$ & $6 \mathrm{~S}$ & $3 \mathrm{C}_{2}$ & $3 \mathrm{C}_{2}$ & $6 \sigma_{\mathrm{d}}$ & \\
\hline $\mathrm{A}_{1}$ & 1 & 1 & 1 & 1 & 1 & $\mathrm{~A}_{1}$ \\
$\mathrm{~A}_{2}$ & 1 & -1 & 1 & 1 & -1 & $\mathrm{~B}_{1}$ \\
$\mathrm{E}$ & 2 & 0 & 2 & 2 & 0 & $\mathrm{~A}_{1}+\mathrm{B}_{1}$ \\
$\mathrm{~F}_{1}$ & 3 & 1 & -1 & -1 & -1 & $\mathrm{E}+\mathrm{A}_{2}$ \\
$\mathrm{~F}_{2}$ & 3 & -1 & -1 & -1 & 1 & $\mathrm{E}+\mathrm{B}_{2}$ \\
\hline
\end{tabular}

Pour les ions ammonium dans la maille on attend donc 30 degrés de liberté (figure 10) qui se répartissent selon;

$$
\Gamma_{\text {total }}\left(N H_{4}^{+}\right)=2 A_{1 g}+A_{2 g}+3 B_{1 g}+B_{2 g}+4 E_{g}+A_{1 u}+3 A_{2 u}+B_{1 u}+2 B_{2 u}+4 E_{u}
$$

On procèdera de la même manière pour dénombrer les degrés de liberté de translation des ions $\mathrm{Br}^{-}$ (corrélation entre $\mathrm{C}_{4 v}$ et $\mathrm{D}_{4 \mathrm{~h}}$ );

$$
\Gamma_{\text {total }}\left(B r^{-}\right)=A_{1 g}+E_{g}+A_{2 u}+E_{u}
$$

Et pour la totalité des 36 degrés de liberté attendus ;

$$
\Gamma_{\text {total }}=3 A_{1 g}+A_{2 g}+3 B_{1 g}+B_{2 g}+5 E_{g}+A_{1 u}+4 A_{2 u}+B_{1 u}+2 B_{2 u}+5 E_{u}
$$

On peut maintenant faire le tri pour les phonons en centre de zone de Brillouin entre les phonons optiques mettant en jeu les modes internes des ions ammonium, les phonons optiques correspondant aux vibrations de réseau et les phonons acoustiques :

$$
\begin{gathered}
\Gamma_{\text {interne }}=2 A_{1 g}+2 B_{1 g}+B_{2 g}+2 E_{g}+A_{1 u}+2 A_{2 u}+2 B_{2 u}+2 E_{u} \\
\Gamma_{\text {externe }}=A_{1 g}+A_{2 g}+B_{1 g}+3 E_{g}+A_{2 u}+B_{1 u}+2 E_{u} \\
\Gamma_{\text {acoustique }}=A_{2 u}+E_{u}
\end{gathered}
$$

\subsection{Un exemple en pratique : la millérite}

La millérite est un cristal de sulfure de nickel (NiS) naturel qui est formé dans des conditions hydrothermales. Ce composé cristallise dans le groupe d'espace $\mathrm{R} 3 \mathrm{~m}$ de groupe ponctuel $\mathrm{C}_{3 v}$ avec $Z=3$ unités formulaires dans la maille primitive rhomboèdrique. La symétrie de site pour Ni et $\mathrm{S}$ est $\mathrm{C}_{\mathrm{s}}$ et le dénombrement des phonons optiques (équation (13)) donne :

$$
\Gamma_{O p t}=3 A_{1}+2 A_{2}+5 E
$$

Les vibrations $\mathrm{A}_{2}$ sont inactives à la fois en Raman et en infrarouge et les vibrations $\mathrm{A}_{1}$ et $\mathrm{E}$ sont actives en infrarouge et en Raman. Les spectres Raman polarisés ont été réalisés sur des monocristaux (aiguilles de petit diamètre $20 \mu \mathrm{m}$ ) nécessitant l'utilisation d'un microscope [3]. Pour les modes actifs en Raman, 
(1) $\mathrm{A}_{1}$

$\mathrm{A}_{2}$

(1) E

(1) $F_{1}$

(3) $F_{2}$
(2) $A_{1}$

(1) $\mathrm{A}_{2}$

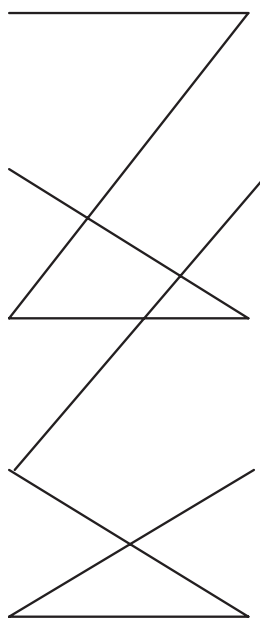

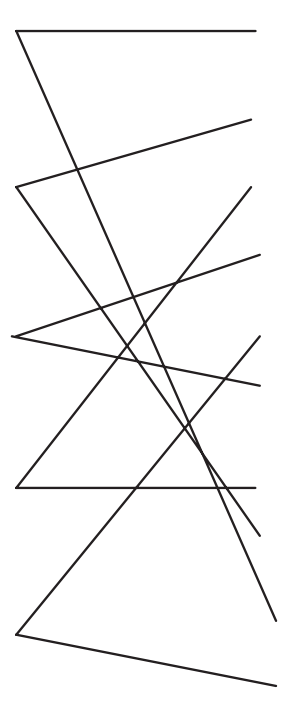

(2) $A_{1 g}$

(1) $A_{2 g}$

(3) $\mathrm{B}_{1 \mathrm{~g}}$

(1) $B_{2 g}$

(4) $E_{g}$

(1) $A_{1 u}$

(3) $A_{2 u}$

(1) $\mathrm{B}_{1 \mathrm{u}}$

(2) $B_{2 u}$

(4) $E_{u}$

Figure 10. Diagramme des corrélations pour $\mathrm{NH}_{4} \mathrm{Br}$ dans sa phase ordonnée. Les chiffres entre parenthèses représentent les modes associés à chaque représentation de l'ion $\mathrm{NH}_{4}^{+}$.

la forme des tenseurs de polarisabilité attendus est la suivante :

$$
\frac{\partial \overleftrightarrow{\alpha}}{\partial q}\left[A_{1}\right]=\left[\begin{array}{lll}
a & 0 & 0 \\
0 & a & 0 \\
0 & 0 & b
\end{array}\right], \quad \frac{\partial \overleftrightarrow{\alpha}}{\partial q}[E]=\left[\begin{array}{lll}
0 & c & d \\
c & 0 & 0 \\
d & 0 & 0
\end{array}\right] \text { et }\left[\begin{array}{lll}
c & 0 & 0 \\
0 & -c & d \\
0 & d & 0
\end{array}\right]
$$

Les spectres polarisés du monocristal ont été obtenus permettant de sonder les composantes xx, xz et zz du tenseur de polarisabilité, c'est-à-dire les modes $\mathrm{A}_{1}$ et E pour une polarisation incidente parallèle à l'axe x du cristal et une analyse de la diffusion selon l'axe $\mathrm{x}$, les modes $\mathrm{E}$ uniquement pour une polarisation incidente parallèle à l'axe $\mathrm{x}$ du cristal et une analyse de la diffusion selon l'axe $\mathrm{z}$ et les modes $A_{1}$ pour une polarisation incidente parallèle à l'axe $\mathrm{z}$ du cristal et une analyse de la diffusion selon l'axe z. Les spectres obtenus (figure 11) permettent une attribution non ambiguë des phonons observés. En effet 3 modes $A_{1}$ sont prévus (équation (25)) et observés (figure 11a), 5 modes E (figure 11b) et bien sûr ces 8 modes sont observés sur la figure 11c.

\section{LA SPECTROSCOPIE DE VIBRATION : TENDANCES ET ÉVOLUTIONS}

Nous venons de voir que la spectroscopie optique est une méthode permettant d'attribuer précisément les phonons optiques en centre de zone de Brillouin grâce aux règles de sélection complémentaires entre l'infrarouge et le Raman et également grâce à la possibilité d'orienter un monocristal par rapport au champ électrique incident (et au champ diffusé). Le point faible des méthodes optiques est lié à la longueur d'onde du rayonnement incident qui ne permet pas, contrairement à la diffusion neutronique, d'explorer les branches de phonons à l'intérieur de la zone de Brillouin. Malgré ce handicap, les avantages sont très nombreux et méritent d'être soulignés.

Tout d'abord les spectromètres optiques sont compacts et économiques, il n'est pas besoin d'investissements coûteux pour équiper un laboratoire et les coûts de fonctionnement sont modestes. 


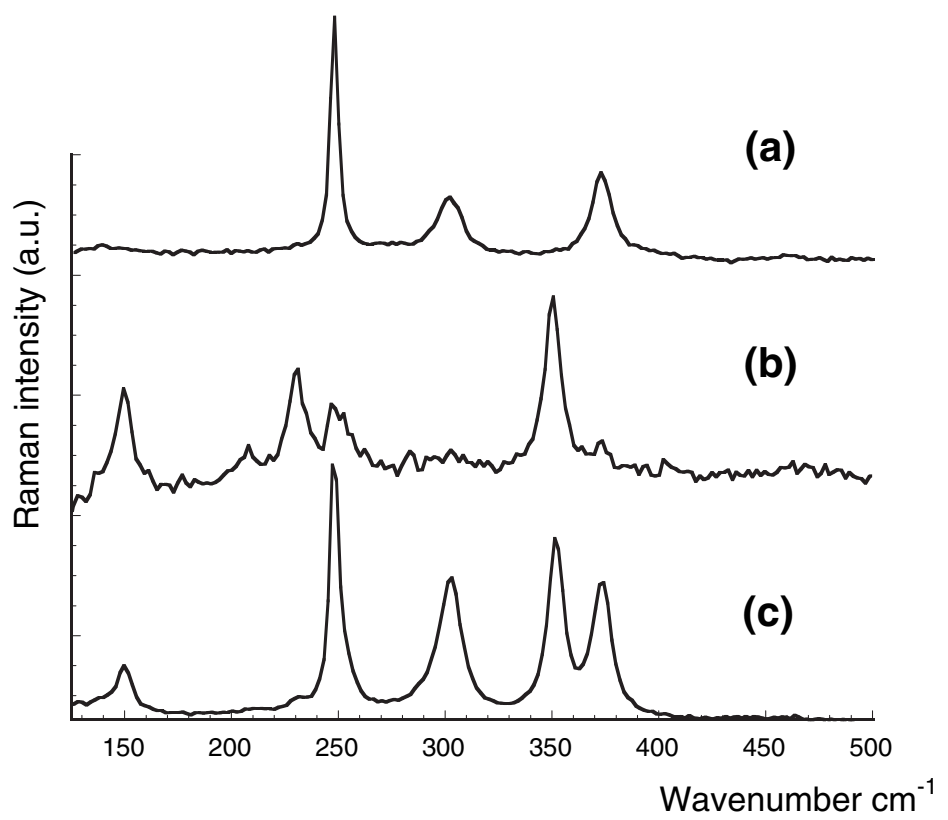

Figure 11. Spectres Raman obtenus dans les trois états de polarisation décrits dans le texte permettant de sonder les composantes zz (a), xz (b) et xx (c) du tenseur de polarisabilité.

Contrairement à la spectroscopie de diffusion des neutrons, la spectroscopie optique ne souffre pas du manque de flux ! Non seulement des petites quantités d'échantillons sont suffisantes pour réaliser en quelques minutes (parfois quelques secondes) des mesures sur une large fenêtre spectrale et avec une bonne résolution $(100 \mu \mathrm{eV})$, mais la plupart des spectromètres infrarouge et Raman sont maintenant couplés à des microscopes permettant ainsi des études sur monocristaux de petites tailles ou des matériaux hétérogènes. Nous allons maintenant présenter brièvement les évolutions récentes de ces techniques et quelques unes de leurs limites.

\subsection{La spectroscopie d'absorption infrarouge}

Du fait de leur compacité les spectromètres IRTF (Infra Rouge à Transformée de Fourier) permettent une grande diversité de montages possibles, pour des mesures en transmission pour des échantillons peu absorbants, des mesures en réflexion (spéculaire, diffuse) et mêmes des couplages avec d'autres techniques. Des difficultés existent cependant, par exemple la nécessité de maintenir les échantillons dans un environnement sec (l'eau absorbe fortement dans l'infrarouge), optimiser le conditionnement des échantillons (pastilles par exemple) pour éviter une trop forte absorption, utiliser des fenêtres optiques transparentes dans l'infrarouge etc. Une autre limitation importante de la spectroscopie IRTF est la grande difficulté pour obtenir des spectres à des nombres d'onde inférieurs à $40 \mathrm{~cm}^{-1}$ environ.

D'énormes progrès ont été réalisés ces dernières années grâce à l'introduction des méthodes de modulation de la polarisation infrarouge [4] permettant d'accroître de manière spectaculaire la sensibilité des mesures pour la spectroscopie de matériaux en couches minces déposées sur un substrat métallique ou diélectrique. Cette technique (PM-IRRAS) permet de réaliser des spectres IRTF polarisés sur des couches d'épaisseur inférieure à $50 \AA$ ! De plus, le montage ne nécessite aucune précaution particulière pour son environnement, les expériences sont réalisées à l'air ambiant. Dans les années 90, ce concept a été généralisé pour des problèmes d'orientations moléculaires dans des films polymères ultra-minces. Encore plus récemment, dans les années 2000, ce principe [5] est exploité 
pour la polarisation circulaire conduisant au développement de la technique du Dichroïsme Circulaire Vibrationnel (VCD) pour les études d'objets chiraux.

\subsection{La spectroscopie de diffusion Raman}

La spectroscopie Raman a connue des développements considérables ces dernières années en raison d'une part de la simplification du montage optique (utilisation d'un filtre Notch pour éliminer la diffusion Rayleigh) et d'autre part de l'exploitation de détecteurs CCD performants. Ainsi les spectromètres Raman sont maintenant systématiquement couplés à des microscopes confocaux ou à des fibres optiques permettant d'étudier des échantillons de petite taille ou placés dans des environnements non conventionnels. Aucun conditionnement spécifique n'est nécessaire et les temps d'acquisition sont très rapides permettant ainsi la réalisation de cartographies d'échantillons hétérogènes, en surface et même en profondeur pour les échantillons transparents. A ce sujet, si le système diffusant possède une bande d'absorption au voisinage de la fréquence d'excitation (par exemple une transition électronique), il en résulte une diffusion Raman de résonance. L'intensité de cet effet Raman résonant peut être de plusieurs ordres de grandeur plus élevé que l'effet Raman ordinaire. La forme du spectre peut aussi être différente : par exemple des harmoniques peuvent être observées avec des intensités appréciables et de nouvelles bandes peuvent apparaitre (modifications des règles de sélection). Le développement des lasers accordables a permis des avancées considérables de cette technique. Les applications importantes de la spectroscopie Raman de résonance concernent les études d'espèces en faibles concentrations ou celles de matériaux très absorbants comme les carbones graphitiques. Les performances aujourd'hui remarquables des spectromètres Raman ont permis aussi le développement de nouvelles méthodes nonlinéaires, en particulier la spectroscopie hyper-Raman [6]. Les règles de sélection sont ici associées au tenseur d'hyper-polarisabilité de rang 3 et sont donc complémentaires de l'infrarouge et du Raman. Comme en infrarouge les techniques de modulation de la polarisation circulaire sont exploitées pour les études de systèmes chiraux («Raman Optical Activity», ROA [7]). Une autre évolution récente est liée à l'intensification du signal Raman d'une molécule située au voisinage d'un nano-objet métallique ou déposée sur un substrat métallique rugueux (effet SERS «Surface Enhanced Raman Spectroscopy»). Cette intensification est due au champ électrique généré par le métal par absorption du rayonnement excitateur (bande plasmon) et qui interagit avec une molécule. Cet effet SERS peut être mis à profit pour obtenir des résolutions spatiales nanométriques [8], en utilisant la pointe métallisée d'un microscope à champ proche (force atomique AFM ou optique NSOM) au voisinage de l'objet à analyser («Tip Enhanced Raman Spectroscopy » TERS). Le rayonnement diffusé par l'échantillon au voisinage de la pointe AFM est détecté et analysé en champ lointain par le spectromètre. La spectroscopie Raman est aujourd'hui une technique incontournable dans le domaine des nanosciences, en particulier pour les études de nanotubes de carbone isolés [9] et même de graphènes [10].

\section{CONCLUSION}

En conclusion nous souhaitons souligner que les spectrométries infrarouge et Raman sont des méthodes puissantes pour l'attribution des phonons optiques en centre de zone de Brillouin. Ces techniques de laboratoire sont légères et pas trop onéreuses et peuvent être couplées à de la microscopie optique ou en champ proche. La rapidité des mesures permet ainsi de faire de l'imagerie, la très haute détectivité (SERS, résonance, modulation de polarisation) l'analyse de films ultra-minces, de nanoobjets, de systèmes à très hautes dilutions. Les progrès considérables de ces dernières années dans les domaines des caméras CCD, des montages optiques et des lasers autorisent aujourd'hui la conception de méthodes innovantes comme le dichroïsme circulaire vibrationnel, l'activité optique Raman ou encore la microscopie hyper-Raman confocale. 
La spectroscopie de vibration «optique» est donc souvent indispensable pour l'attribution des phonons optiques et est donc un préliminaire incontournable à des études par diffusion neutronique. Elle offre également des informations inaccessibles actuellement par d'autres moyens.

\section{Références}

[1] Plateforme "Spectroscopie et Imagerie Vibrationnelle" de l'Institut des Sciences Moléculaires à l'Université Bordeaux 1.

[2] R. Currat, article dans ce volume.

[3] F. Guillaume, S. Huang, K.D.M. Harris, M. Couzi, D. Talaga, J. Raman Spectrosc., 39: 14191422, 2008

[4] T. Buffeteau, B. Desbat, J.M. Turlet, Appl. Spectrosc. 45 (3) 380 (1991)

[5] T. Buffeteau, F. Lagugne-Labarthet, C. Sourisseau, Appl. Spectrosc. 59 (6) 732 (2005)

[6] V. Rodriguez, D. Talaga, F. Adamietz, JL. Bruneel, M. Couzi, Chem. Phys. Lett. 431 (1-3) 190 (2006)

[7] L. Barron «Molecular Light Scattering and Optical Activity », $2^{\mathrm{d}}$ Edition, Cambridge University Press, Cambridge (2004)

[8] A. Hartschuh, E.J. Sanchez, X.S. Xie, L. Novotny, Phys. Rev. Lett., 90 (9) 095503 (2003)

[9] J.L. Sauvajol, E. Anglaret, S. Rols, L. Alvarez, Carbon, 40 (10) 1697 (2002)

[10] A.C. Ferrari, J. Meyer, C. Scardaci, C. Casiraghi, M. Lazzeri, F. Mauri, Phys. Rev. Lett., 855214 (2007) 